\title{
Medical Image Segmentation by Evolutionary Approach and Watershed Morphology
}

\author{
Ahmad El Allaoui \\ Labo Matsi, Esto, B.P 473, University Mohammed I \\ Oujda, Morocco.
}

\begin{abstract}
Segmentation by watershed transform is a fast, robust and widely used in image processing and analysis, but it suffers from over-segmentation. We present in this paper some improvements to this algorithm based on the evolutionary algorithm and mathematical morphology in order to get over this difficulty. The performance of this method is validated on medical images. The results obtained show the good performance of this approach.
\end{abstract}

\section{General Terms}

Medical image segmentation, over-segmentation, markers controlled watershed. Morphological dilatation.

\section{Keywords}

Image processing, medical image segmentation, Watershed, Evolutionary algorithm, dilatation, Mathematical morphology.

\section{INTRODUCTION}

The segmentation is a very important stage in images and interpretation processing. There are two main approaches to segmentation: the frontier approach and the region approach $[18,19]$. The segmentation by watershed combine the two approaches $[1,2]$.This is a powerful technique for rapid detection of both edges and regions.

The major problem of the watershed transform is oversegmentation. Indeed, this algorithm is sensitive to any local minimum in the image, and tends to define the lines of the watershed transform where each local minimum gives rise to a region. To avoid this problem, powerful tools adapted to different problems have been proposed in the literature [3, 7]:

- Either reduce the number of minima and avoid calculation of too many regions.

- Proceed by either filtering techniques by merging the regions according to similarity criteria after spectral and spatial application of the watershed.

We have used markers to reduce the number of regional minima.

Considering the great success of the evolutionary algorithms in the optimisations problems, we exploit them to get over the over-segmentation. We have presented an algorithm of segmentation by evolutionary watershed $(E W)$.

In Section 2 image segmentation with watershed algorithm is presented. Section 3 gives an introduction to evolutionary algorithm. The evaluation criterion of segmentation is detailed in the next section. The proposed evolutionary watershed noted $(E W)$ is presented in section 5 . In section 6 , a validation of our approach is given; experimental results are obtained by medical images. Finally we give a conclusion.

\section{WATERSHED ALGORITHM}

The watershed transform is a morphological tool for image segmentation. It is proposed by Digabel et Lantuejoul [1, 2, 4], which consider a grey level image as a topographic relief.

\author{
M'barek Nasri \\ Labo Matsi, Esto, B.P 473, University Mohammed I \\ Oujda, Morocco.
}

If one combines the grey level of each point at an altitude. It is then possible to define the watershed transform as the ridge forming the boundary between two watersheds. This is to compute the watershed of the said relief. Watersheds thus obtained correspond to regions of the image.

Watershed represents the boundaries between adjacent catchments. The minimum can be interpreted as markers of watershed regions and the watershed can be interpreted as contours, figure 1 .

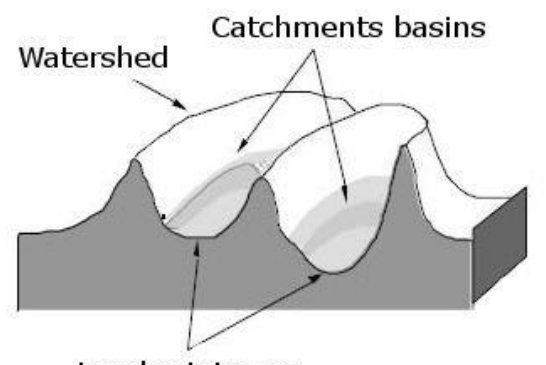

Local minimum

Fig 1: Modelling of contours by watershed transform

Many pre-processing techniques are proposed by the different researchers (filters, morphological operators) were then designed to reduce the number of regions not significant. Nevertheless, the work of Beucher $[1,2]$ demonstrated the limitations of these methods namely the over-segmentation.

\subsection{The over-segmentation.}

The use of the single watershed algorithm does not really good segmentation because far too many regions are detected, figure 2 . There are two main methods to limit this oversegmentation:

- hierarchical watershed segmentation $[1,2,9]$.

- and watershed by markers $[3,10,11]$.

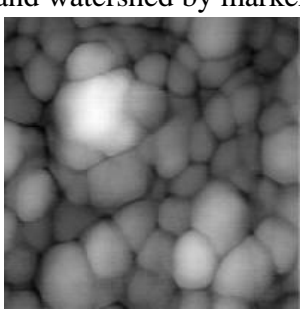

Original image

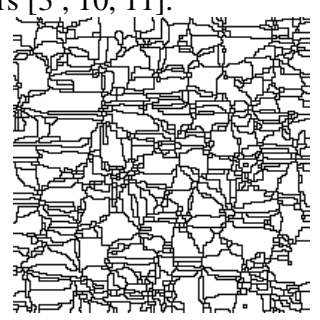

Watershed, 715regions
Fig 2: Over-segmentation

The hierarchical segmentation: The hierarchical approach is to generate a tree of regions from the result of the watershed. Regions and watershed are first indexed, and then the process of hierarchical segmentation process merges the regions whose borders are the lowest. The result is a tree in which it is possible to explore the different levels of fusion regions $[1,2$, 
$6,8,9]$. Several studies have been made to get over the problem of over-segmentation by markers $[1,3,5,7,10,11]$.

\subsection{Mathematical morphology}

Mathematical morphology is a nonlinear branch of the signal processing field and concerns the application of set theory concepts to image analysis. Morphology refers to the study of shapes and structures from a general scientific perspective. Morphological filters or operators are nonlinear transformations, which modify geometric features of images.

These operators transform the original image into another image through the iteration with other image of a certain shape and size which is known as structuring element [10].

The structuring element is a basic structure that will be used to analyze locally, by comparison, the shapes of interest.

The choice of shape and size of the structuring element is very important: it influences the result [10], it may take the form of a disk of radius $r$, a rectangle or other shape.

There are several morphological tools in image segmentation namely erosion, dilation, reconstruction etc. We are interested in this work in the morphological dilation.

\subsubsection{Morphological dilatation}

Let $X$ be a subset of $E$. If with any $x$ we associate a position $B(x)$ of the structuring element $\mathrm{B}$, then the set $\mathrm{X}$ dilated by the structuring element $\mathrm{B}$ is defined as following:

$$
\delta_{B}(X)=\{x \in E, B(x) \cap X \neq \phi\}
$$

The structuring element $B$, denoted by its center is moved to occupy successively all positions of the space $E$. For each position, we ask the question: does $B$ intersects at $X$ ? Positive responses are all dilated, figure 3 .

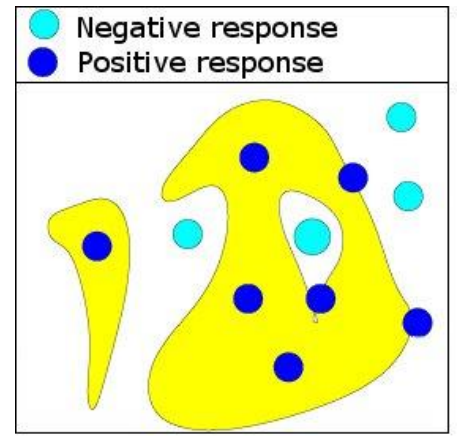

Fig 3: Morphological dilatation.

The dilatation is characterized by the following properties:

- $\quad$ Fills holes smaller than $B$.

- Fills narrow channels.

- Welds both forms close.

- The size of objects increases.

- Small details disappear.

In what follows, we propose a marker-based watershed on mathematical morphology and evolutionary strategies.

\section{EVOLUTIONARY STRATEGIES}

Evolutionary strategies $(E S)$ are particular methods for optimizing functions. These techniques are based on the evolution of a population of solutions which under the action of some precise rules optimize a given behavior, which initially has been formulated by a given specified function called fitness function [16].

An ES algorithm manipulates a population of constant size. This population is formed by candidate points called chromosomes. Each of the chromosomes represents the coding of a potential solution to the problem to be solved, it is formed by a set of elements called genes, these are reals[17].

At each iteration, called generation, is created a new population from its predecessor by applying the genetic operators: selection and mutation. The mutation operator perturbs with a Gaussian disturbance the chromosomes of the population in order to generate a new population permitting to further optimize the fitness function.

This procedure allows the algorithm to avoid the local optimums. The selection operator consists of constructing the population of the next generation. This generation is constituted by the pertinent individuals $[16,17]$

Figure 4 illustrates the different operations to be performed in a standard $E S$ algorithm [16]:

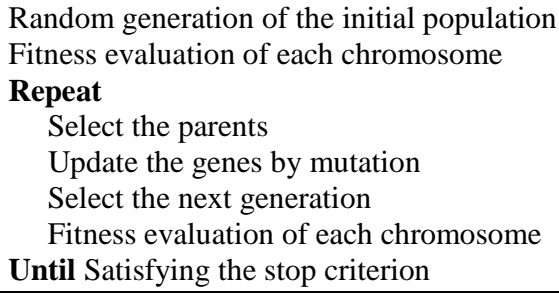

Fig 4: Standard SE algorithm.

\section{EVALUATION OF A SEGMENTATION}

There are a multitude of segmentation methods whose effectiveness is difficult to assess. Currently, some work has been done to solve the general problem of evaluating a segmentation result $[12,13]$. There are two types of evaluation approaches [14].

The first evaluates the quality of a segmentation result by measuring its similarity to reference segmentation. Several studies have been made in medical imaging [15].

The second is based on unsupervised evaluation criteria for estimating the quality of a segmentation result from statistics calculated for each detected region.

There are several criteria for evaluating unsupervised segmentation [14]. The value of each criterion increases with the quality of the segmentation result [14].

A criterion value close to 1 indicates a very good result of segmentation. Different criteria are described in detail in [13], we are interested in this work in the criterion of Levine and Nazif.

\subsection{Inter-region contrast of Levine and Nazif}

This criterion computes the sum of contrasts of regions $\left(R_{i}\right)$ weighted by their area $\left(A_{i}\right)$. The contrast of a region is defined from the existing contrasts with regions that are contiguous.

$$
\begin{gathered}
C_{I R}=\frac{\sum_{R_{i}} A_{i} c_{i}}{\sum_{R_{i}} A_{i}} \\
\text { Where } c_{i}=\sum_{R_{j}} \frac{l_{i j}\left|m_{i}-m_{j}\right|}{l_{i}\left(m_{i}+m_{j}\right)}
\end{gathered}
$$

$m_{i}$ mean of the region $R_{i} ; l_{i j}$, length of the common border between $R_{i}$ and $R_{j} ; l_{i}$, perimeter of the region $R_{i}$. 


\section{PROPOSED SEGMENTATION}

We have developed a segmentation method based on evolutionary strategies, and watershed noted $E W$.

\subsection{Proposed coding}

The $E W$ algorithm consists of selecting among all of the possible partitions the optimal partition by maximizing a criterion for validating segmentation. This leads to identify region in the image markers $(P s) \quad 1 \leq s \leq C$ and using the structuring element $S$ in morphological gradient and in the morphological dilation. Thus we suggest the real coding as:

$$
\begin{aligned}
& \operatorname{chr}=\left(P_{s j}, S_{k}\right)_{1 \leq s \leq C, 1 \leq j \leq N, 1 \leq k \leq M} \\
& =\left(P_{11} . . P_{1 N} P_{21} . . P_{2 N} . . P_{s 1} . . P_{s N} . . P_{C 1} . . P_{C N}, S_{1} . . S_{M}\right)
\end{aligned}
$$

The chr chromosome is a real line vector of dimension $C \times N+M . P_{\mathrm{sj}}$ is formed by coordinate $(x, y)$ of this point, then $N=2, P_{s}\left(x_{s}, y_{s}\right)$.

The structuring element $S$ is a positive integer greater than 2 . Example: $C=2 ; N=2 ; M=1$

$$
C h r=\left(P_{1}, P_{2}, S\right)=\left(x_{1}, y_{1}, x_{2}, y_{2}, S_{1}\right)
$$

\subsection{The proposed fitness function}

Let $c h r$ be a chromosome of the population formed by the point $\left(P_{s}\right)_{1 \leq s \leq C}$, for computing the fitness function value associated with $c h r$, we define the fitness function $C_{R E}(c h r)$ (inter-region contrast of Levine and Nazif) presented in paragraph (4.1) which expresses the behavior to be optimized. The chromosome $c h r$ is optimal if $C_{I R}(c h r)$ is maximal.

\subsection{The proposed $\mathrm{EW}$ algorithm}

Figure 5 shows the different steps of the proposed evolutionary watershed $E W$ algorithm.

\begin{tabular}{l} 
Stage 1: \\
1. Fix: \\
- The size of the population maxpop. \\
- The maximum number of generation maxgen. \\
- The number of region $C=2$. \\
\hline
\end{tabular}

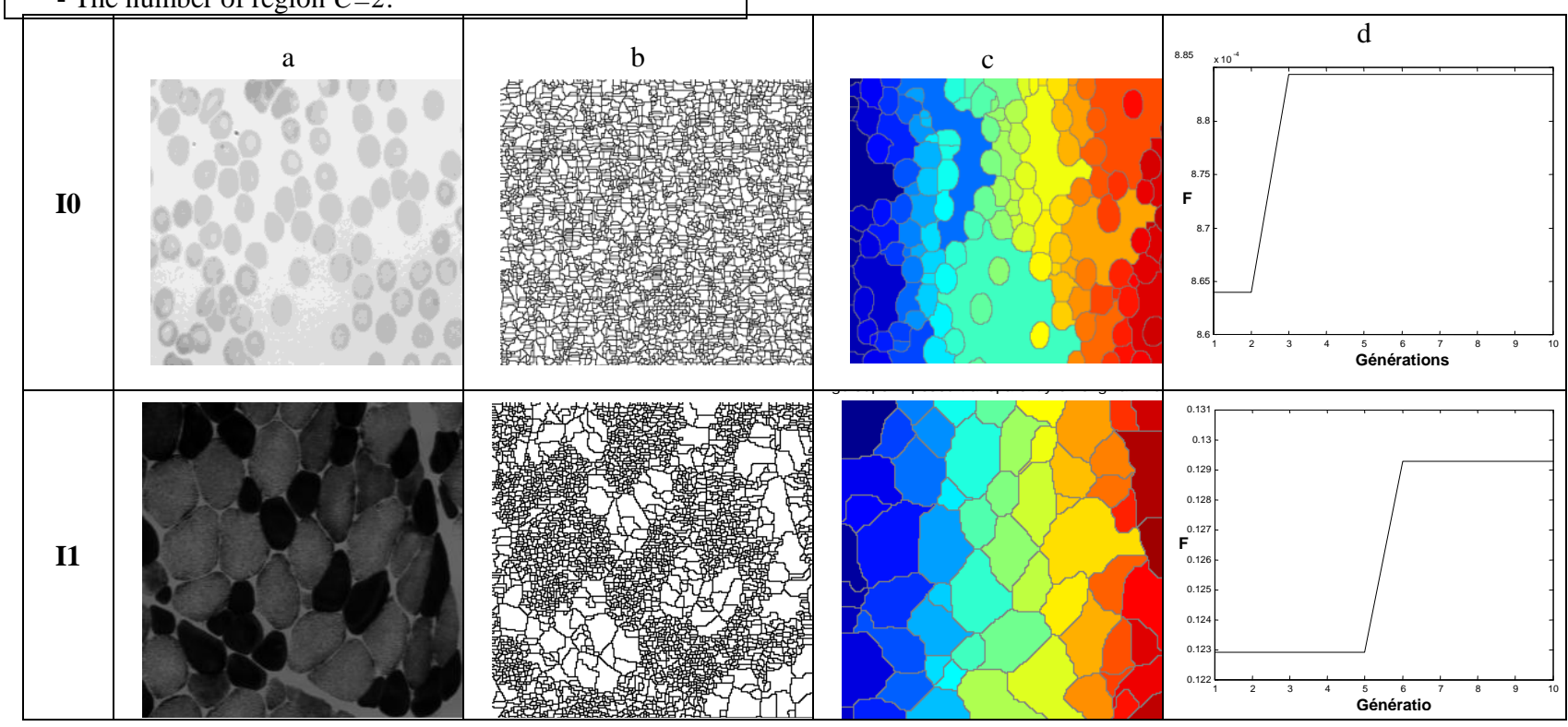

2. Read the original image $I$.

3. Gradient of the $I$.

4. Generate randomly the population $P$ : $P=\left\{c h r_{l}, . ., c h r_{k}, \ldots, c h r_{\text {maxpop }}\right\}$

5. Compute for each $c h r$ of $P$ its fitness value $C_{I R}(c h r)$

Stage 2:

Repeat

1. Order the chromosomes chr in $P$ from the best to the poor ( in an increasing order of $C_{I R}$ ).

2. Choose the best chromosomes chr

3. Generate randomly the constant $f_{m}\left(f_{m} \in[0.5,1]\right)$

4. Mutation of all the chromosomes chr of $P$ except the first one (elitist technique): $g{ }_{s}=g_{s}+f_{m} \times N(0,1)$

5. Marking of the image points of $c h r$, following by dilatation (marker for regions).

6. Expand and imposed markers

7. Compute the watershed transform

8. Compute for each $c h r$ of $P$ its fitness value $C_{I R}(c h r)$

Until $N b \_g e n$ (generation number) maxgen

Fig 5: The proposed EW algorithm

\section{EXPERIMENTALE RESULTS}

\subsection{Introduction}

In order to evaluate the performances of the proposed method we have considered 5 grey level medical images denoted respectively $I 0, I 1, I 2, I 3$ and $I 4$. We have compared the results obtained by our method with those obtained by watershed without markers.

\subsection{Results of segmentation}

Figure 6 and table 1 shows the results segmentation.

a. initial image $a$,

b. watershed applied to the gradient of image $a$,

c. results of $E W$ segmentation

d. Fitness value with respect to the generation 


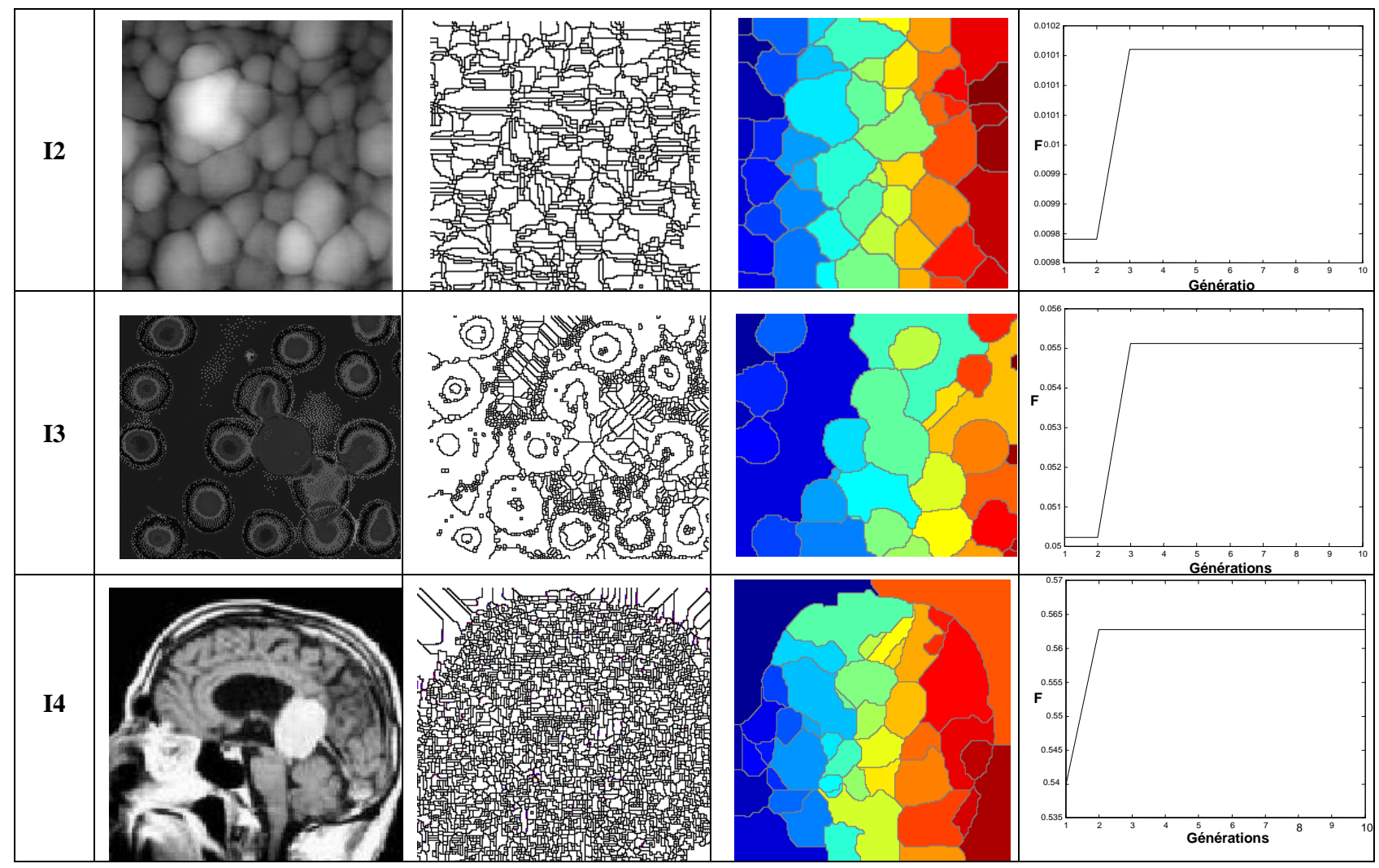

Fig 6: Results of segmentation.

Table1 : Information results

\begin{tabular}{|c|c|c|c|c|c|}
\hline Image & 10 & II & $I 2$ & I3 & I4 \\
\hline Value of structuring element $S$ obtained by $E W$ & 6 & 9 & 7 & 3 & 9 \\
\hline Number of regions without markers & 2772 & 715 & 3253 & 3160 & 164503 \\
\hline Number of regions obtained by $E W$ & 135 & 62 & 57 & 32 & 46 \\
\hline$C_{R I}$ & 0.8844 & 0.1293 & 0.0102 & 0.0551 & 0.5628 \\
\hline
\end{tabular}


The proposed method is applied to 5 medical images (Xray, MRI): cell, muscle and brain. The proposed algorithm converges quickly and no more than 6 iterations in all images $I 0$ to $I 4$, figure 6 (d). Indeed the optimal chromosome obtained by $E W$ is according to its fitness value calculated by the criterion of Levine and Nazif, table1. This chromosome is the best segmentation. It consists of markers of the image and the structuring element used in the gradient and the morphological dilation.

The results of segmentation by the proposed algorithm $E W$ shows clarity and detecting objects (contours and region) the problem of local minima and the choice of the structuring element is eliminated. The problem of oversegmentation is solved.

The algorithm is useful to segment the objects that touch each other in an image.

\section{CONCLUSION}

In this paper, we have proposed a method of segmentation by watershed and evolutionary algorithm. We have calculated automatically region markers and structuring element. The markers are used to control the watershed to obtain good results.

The markers are generated automatically positioned and thereby avoiding the problem of over-segmentation, and manual marking.

\section{REFERENCES}

[1] S. Beucher, Unbiased Implementation of the Watershed Transformation based on Hierarchical Queues. CMM Internal note, Paris 206 School of Mines, 2004.

[2] S. Beucher. Watershed, hierarchical segmentation and waterfall algorithm. Mathematical morphology and its applications to image processing, pages 6976. Kluwer Academic Publishers, 1994.

[3] M. Ali HAMDI. Modified Algorithm markercontrolled watershed transform for Image segmentation Based on Curvelet Threshold. Canadian Journal on Image Processing and Computer Vision. pp, 88-91. Vol. 2 No. 8, December 2011.

[4] J. Serra, Image Analysis and Mathematical Morphology. Academic Press, London 1982.

[5] R. C. Gonzalez and R. E. Woods, Digital Image Processing, Prentice-Hall, Upper Saddle River, NJ, USA, 2nd edition, 2002.

[6] Vincent, L., "Morphological Grayscale Reconstruction in Image Analysis: Applications and Efficient Algorithms," IEEE Transactions on Image Processing, Vol. 2, No. 2, pp. 176-201, April, 1993.

[7] Sarah Ghandour. Segmentation d'images couleurs par morphologie mathématique : application aux images microscopiques. Thèse doctorat, Université Paul Sabatier, Toulouse 12 Juillet 2010.

[8] P. Arbeláez and L. Cohen. Constrained image segmentation from hierarchical boundaries. CVPR, 2008.

[9] P. Arbeláez, M. Maire, C. Fowlkes and J. Malik.Contour Detection and Hierarchical Image Segmentation IEEE TPAMI, Vol. 33, No. 5, pp. 898-916, May 2011.
[10] K. Parvati, et al, Image Segmentation Using GrayScale Morphology and Marker-Controlled Watershed Transformation. Discrete Dynamics in Nature and Society, vol. 2008, Article ID 384346, 8 pages, 2008.

[11] S. Lefèvres. Segmentation par Ligne de Partage des Eaux avec Marqueurs Spatiaux et Spectraux. XXIIe colloque GRETSI (traitement du signal et des images), Dijon (FRA), 8-11 septembre 2009.

[12] V. Letournel. Contribution à l'évaluation d'algorithmes de traitement d'images. Thèse de Doctorat de l'ENST. 2002.

[13] S. Philipp-Foliguet. Evaluation de la segmentation. Rapport Technique, 2001.

[14] C. Rosenberger, S. Chabrier, H. Laurent, B. Emile. Unsupervised and supervised image segmentation evaluation. Chapitre 18, pages 365-393 du livre Advances in Image and Video Segmentation, Pr. Yu-Jin Zhang, Idea Group Publishing, 2006.

[15] J. Brunette, R. Mongrain et G. Cloutier. A novel realistic three-layer phantom for intravascular ultrasound imaging. International Journal Cardiac Imaging, 17(5),pp 371- 381 (2001).

[16] M. Nasri. Contribution à la classification de données par Approches Evolutionnistes : Simulation et application aux images de textures. Thèse de doctorat. Université Mohammed premier Oujda 2004.

[17] J.M. Renders. Algorithmes génétiques et Réseaux de Neurones. Editions HERMES, 1995.

[18] A. EL Allaoui, M. Merzougui, M. Nasri, M. EL Hitmy and $H$. Ouariachi. Evolutionary Image Segmentation By Pixel Classification Application To Medical Images. IJCIIS International Journal of Computational Intelligence and Information Security, ISSN: 1837-7823, Vol. 2, No. 3 pp. 12-24. March 2011.

[19] A. EL Allaoui, M. Nasri, M. Merzougui , M. EL Hitmy et B. Bouali. Medical Image Segmentation By Region Evolutionary Approach. The 3rd International Conference on Multimedia Computing and Systems ICMCS'12 Tangier, CDROM, 10-12 Mai 2012.

\section{AUTHOR PROFILE}

Ahmad EL ALLAOUI works on image processing and pattern recognition in MATSI laboratory of the school of Technology, University of Oujda, Morocco. He has his master in the field of computer sciences at University the Med Benabdellah at Fez. His main field of research interest is segmentation of medical image based on new methods (genetic and evolutionary approaches, neural networks, fuzzy logic).

M'barek Nasri is a senior lecturer at the School of technology of Oujda University since 1996. He obtained his Ph.D degree in 2004 in the field of data Classification from the University of Oujda, department of physics. He obtained the habilitation degree in 2006 from the same university. His field of research interest is in image processing and its application to the quality control by artificial vision and medical imaging. MATSI laboratory of the school of Technology, University of Oujda, Morocco. 